TRANSACTIONS OF THE

AMERICAN MATHEMATICAL SOCIETY

Volume 357, Number 10, Pages 4131-4140

S 0002-9947(04)03706-7

Article electronically published on December 10, 2004

\title{
NONUNIFORM HYPERBOLICITY FOR SINGULAR HYPERBOLIC ATTRACTORS
}

\author{
WILMER J. COLMENÁREZ RODRIGUEZ
}

\begin{abstract}
In this paper we show nonuniform hyperbolicity for a class of attractors of $C^{2}$ flows in dimension three. These attractors are partially hyperbolic with central direction being volume expanding, contain dense periodic orbits and hyperbolic singularities of the associated vector field. Classical expanding Lorenz attractors are the main examples in this class.
\end{abstract}

\section{INTRODUCTION}

In the category of smooth flows, uniform hyperbolicity fails e.g. when some hyperbolic singularity of the associated vector field is accumulated by hyperbolic periodic orbits inside an attractor. This phenomena implies at first glance some partial hyperbolicity of the system. The class of attractors we are dealing with have singularities (hyperbolic ones) and are partially hyperbolic with volume expanding central direction. We show that if such an attractor has dense periodic orbits, then every orbit outside an unstable manifold of some singularity is nonuniformly hyperbolic. These attractors were studied in MPP in connection with a robust transitive property (also see [MP), and one main and motivating example is the so-called expanding or classical Lorenz attractor $[\mathrm{G}]$. We hope the result in this paper is a first step to giving a proof of the existence of an SRB measure for such attractors in another work already in progress (see $[\bar{V}]$ for a general account about SRB measures).

Now, we set the precise definitions of the objects and properties mentioned above. Let $X_{t}$ denote a $C^{r}$ flow, $r \geqslant 2$ on a Riemannian 3-manifold $M$ with generating vector field $X(q)=\left.(d / d t) X_{t}(q)\right|_{t=0}$. A compact invariant set $\Lambda \subset M$ is said to be an attractor if for some neighborhood $U \supset \Lambda$ we have $\Lambda=\bigcap_{t \geqslant 0} X_{t}(U)$ (such an $U$ is called a trapping region of $\Lambda$ ), and $\left.X_{t}\right|_{\Lambda}$ is topologically transitive, that is, $\Lambda$ is the $\omega$-limit set of some orbit. We say that $\Lambda$ is partially hyperbolic if there is a continuous invariant splitting $T_{\Lambda} M=E_{\Lambda}^{s} \oplus E_{\Lambda}^{c}$ where the stable subbundle $E_{\Lambda}^{s}$ is one-dimensional, the central subbundle $E_{\Lambda}^{c}$ is two dimensional, contains the flow direction $E_{\Lambda}^{X}$ and there are positive constants $\lambda, c$ such that, for every $t \geqslant 0$,

$$
\left\|\left.D X_{t}\right|_{E_{\Lambda}^{s}}\right\| \leqslant c e^{-\lambda t} \quad\left(E_{\Lambda}^{s} \text { is contracting }\right)
$$

Received by the editors June 17, 2003 and, in revised form, December 2, 2003. 2000 Mathematics Subject Classification. Primary 37Dxx; Secondary 37C15.

Key words and phrases. Nonuniform hyperbolicity, singular attractors, 3-flows.

This work was partially supported by FONACIT and CDCHT-UCOLA (Venezuela) and by CNPq (Brazil). 
and

$$
\left\|\left.D X_{t}\right|_{E_{\Lambda}^{s}}\right\| \cdot\left\|\left.D X_{-t}\right|_{E_{\Lambda}^{c}}\right\| \leqslant c e^{-\lambda t} \quad\left(E_{\Lambda}^{c} \text { is dominated by } E_{\Lambda}^{s}\right) .
$$

As in [MPP], a singular hyperbolic attractor is a partially hyperbolic attractor containing singularities, all of them hyperbolic, and whose central direction $E_{\Lambda}^{c}$ is volume expanding:

$$
\left|\operatorname{det}\left(\left.D X_{t}\right|_{E_{\Lambda}^{c}}\right)\right| \leqslant c e^{\lambda t} \quad \text { for } t<0 .
$$

For linear spaces or vector subbundles $E_{1}$ and $E_{2}$ of $T M$ we denote $\measuredangle\left(E_{1}, E_{2}\right)$ the angle between $E_{1}$ and $E_{2}$. According to $\left[\underline{S}\right.$, Chapter 6 , p.110, the orbit $\mathcal{O}_{X}(p)=$ $\left\{X_{t}(p): t \in \mathbb{R}\right\}$ of a point $p \in M$ under the action of a flow $X_{t}$ is said to be nonuniformly hyperbolic if for each $t \in \mathbb{R}$ there exists a continuous splitting

$$
T_{X_{t}(p)} M=E_{X_{t}(p)}^{s} \oplus E_{X_{t}(p)}^{u} \oplus E_{X_{t}(p)}^{X}
$$

which is invariant by the differential $D X_{s}, s \in \mathbb{R}$, and there is a positive real number $\eta$ such that for each $\varepsilon>0$ small enough there exists a continuous positive function $C_{\varepsilon}$ along $\mathcal{O}_{X}(p)$ satisfying:

(1) $C_{\varepsilon}\left(X_{t}(p)\right) \leqslant e^{\varepsilon|t|} C_{\varepsilon}(p)$ for every $t \in \mathbb{R}$.

(2) $\measuredangle\left(E_{X_{t}(p)}^{s}, E_{X_{t}(p)}^{u}\right), \measuredangle\left(E_{X_{t}(p)}^{s, u}, E_{X_{t}(p)}^{X}\right) \geqslant C_{\varepsilon}\left(X_{t}(p)\right)^{-1}$ for every $t \in \mathbb{R}$.

(3) $E_{X_{t}(p)}^{u}$ is nonuniformly expanded by $D X_{s}$; that is, for $s, t \in \mathbb{R}$ with $s>0$, we have

$$
\left\|D X_{s}\left(X_{t}(p)\right) v\right\| \geqslant C_{\varepsilon}^{-1}\left(X_{t+s}(p)\right) e^{\eta s}\|v\|, \text { for } v \in E_{X_{t}(p)}^{u} .
$$

(4) $E_{X_{t}(p)}^{s}$ is nonuniformly contracted by $D X_{s}$; i.e., for $s, t \in \mathbb{R}$ with $s>0$, we have

$$
\left\|D X_{s}\left(X_{t}(p)\right) v\right\| \leqslant C_{\varepsilon}\left(X_{t+s}(p)\right) e^{-\eta s}\|v\|, \text { for } v \in E_{X_{t}(p)}^{s} .
$$

If the function $C_{\varepsilon}$ is constant, we call $\mathcal{O}_{X}(p)$ an (uniformly) hyperbolic orbit. A compact invariant subset of $M$ is said to be (uniformly) hyperbolic if every orbit inside it is hyperbolic and the respective constants are uniform on every such orbit.

Let $\operatorname{sing}(X \mid \Lambda)$ denote the set of singularities of the vector field $X$ contained in $\Lambda$. If $\Lambda$ is singular hyperbolic, then $\operatorname{sing}(X \mid \Lambda)$ is a nonempty subset of $\Lambda$ consisting of hyperbolic singularities, all of them Lorenz as in MPP. In particular, $\operatorname{sing}(X \mid \Lambda)$ is finite. Define $\Lambda^{*}=\Lambda \backslash W^{u}(\operatorname{sing}(X \mid \Lambda))$, where $W^{u}(\operatorname{sing}(X \mid \Lambda))$ denotes the union of unstable manifolds of elements in $\operatorname{sing}(X \mid \Lambda)$.

The usual approach for nonuniform hyperbolicity starts with the existence a priori of some invariant probability measure with support contained in $\Lambda$. Then, some notions of orbit regularity as well as expansion (resp. contraction) rates are introduced for a subset with full measure. Then, Oseledec's Theorem applies to guarantee the existence of a subset $\Lambda$ with full measure on which the flow is nonuniformly hyperbolic (see, e.g. [O, $[\mathrm{P},[\mathrm{PS},[\mathrm{KM}]$ ).

Singular hyperbolicity and $\operatorname{dim}(M)=3$ imply that some large set $\Lambda^{*}$ of orbits in $\Lambda$ is nonuniformly hyperbolic. More precisely, the condition of uniform expansion volume implies that the orbits in $\Lambda^{*}$ are regular in the Lyapunov sense and the corresponding Lyapunov exponents

$$
\chi(x, v)=\limsup _{t \rightarrow+\infty} \frac{1}{t} \log \left\|D X_{t}(x) \cdot v\right\|
$$

are nonzero for $v \notin\{s \cdot X(x): s \in \mathbb{R}\}$ (see definitions in $[\underline{\mathrm{P}}]$ ). 
We shall prove nonuniform hyperbolicity for orbits in the invariant (and no compact) set $\Lambda^{*}$. But we follow a direct approach that does not require the existence a priori of some invariant probability measure with large support, instead, we use denseness of periodic orbits in $\Lambda$.

Our result is the following:

Theorem A. Let $\Lambda$ be a singular hyperbolic attractor for a $C^{2}$ vector field on a three-dimensional manifold. Suppose that $\Lambda$ contains dense periodic orbits. Then, every orbit in $\Lambda^{*}$ is nonuniformly hyperbolic.

It is clear from the definition that $\Lambda^{*}$ is a residual set in $\Lambda$.

Theorem $\mathrm{B}$ below is a precise reformulation of Theorem $\mathrm{A}$ according to the definition of nonuniformly hyperbolic orbit and preliminary facts above. This result will be proved in the next section following some ideas of the proof of Oseledec's Theorem [O] given in $[\mathrm{M}]$ and uses estimates in $[\mathrm{KM}]$ and Lemma 1 from [MPP.

Theorem B. Let $\Lambda$ be a singular hyperbolic attractor with dense periodic orbits. Then, for every $p \in \Lambda^{*}$ the central space along the orbit $\mathcal{O}_{X}(p)$ of $p$ admits a continuous $D X_{s}$-invariant splitting $E_{X_{t}(p)}^{c}=E_{X_{t}(p)}^{X} \oplus E_{X_{t}(p)}^{u}, s, t \in \mathbb{R}$, and there is a positive $\eta$ so that for each sufficiently small $\varepsilon>0$, there is a positive continuous function $C_{\varepsilon}$ on $\mathcal{O}_{X}(p)$ such that

(i) $C_{\varepsilon}\left(X_{t}(p)\right) \leqslant e^{\varepsilon|t|} C_{\varepsilon}(p)$ for every $t \in \mathbb{R}$;

(ii) $E_{X_{t}(p)}^{u}$ is nonuniformly expanded by $D X_{s}$; that is, if $s, t \in \mathbb{R}$ and $s>0$, then

$$
\left\|D X_{-s}\left(X_{t}(p)\right) v\right\| \leqslant C_{\varepsilon}\left(X_{t}(p)\right) e^{-\eta s}\|v\|, \text { for } v \in E_{X_{t}(p)}^{u},
$$

(iii) $\measuredangle\left(E_{X_{t}(p)}^{u}, E_{X_{t}(p)}\right) \geqslant C_{\varepsilon}\left(X_{t}(p)\right)^{-1}$ for every $t \in \mathbb{R}$.

We shall give the proof of Theorem $\mathrm{A}$ in Section 2 as an immediate consequence of Theorem B. The proof of Theorem B will be given in Section 3 First, in the next section, we present some preliminary facts.

The author thanks the referee for corrections and also thanks C. A. Morales and M. J. Pacifico for helpful comments on an earlier version of this paper.

\section{Preliminaries}

Let $\Lambda \subset M$ be a singular hyperbolic attractor for a 3-flow $\left\{X_{t}\right\}$ on $M$ generated by a $C^{r}$ vector field $X, r \geqslant 1$. Let $U \subset M$ be a trapping region of $\Lambda$. Let $E_{\Lambda^{*}}^{c}$ denote the restriction to $\Lambda^{*}$ of the center subbundle $E_{\Lambda}^{c}$ (we use consistently this sort of notation through this paper). For any map $g$ and any subset $A$ of the domain of $g$ we denote $\left.g\right|_{A}$ the restriction of $g$ to $A$ and also denote $I d_{E}$ the identity map restricted to any subset $E$.

We recall the linear Poincaré flow studied in [D]. Let $\mathcal{R}=\Lambda \backslash \operatorname{sing}(X \mid \Lambda)$ be the set of regular points of $\Lambda$. For each $p \in \mathcal{R}$ let $E_{p}=\{\alpha X(p): \alpha \in \mathbb{R}\}$ be the flow direction at $p$, and let $E_{p}^{\perp}$ denote the orthogonal complement of $E_{p}$ in $T_{p} M$ of $E_{p}$. We define $E_{\mathcal{R}}$ and $E_{\mathcal{R}}^{\perp}$ as the subbundles of $T_{\mathcal{R}} M$ with fibers $E_{p}$ and $E_{p}^{\perp}$ over $p \in \mathcal{R}$ respectively and provide the norms induced from the Riemannian one in $T_{p} M$. Thus, the one-dimensional subbundle $F_{\mathcal{R}}$ with fiber $F_{p}=E_{p}^{\perp} \cap E_{p}^{c}$ over $p \in \mathcal{R}$ is complementary to $E_{\mathcal{R}}$ in $E_{\mathcal{R}}^{c}$. Let $\pi_{p}=\pi_{E_{p}^{\perp}}: T_{p} M \rightarrow E_{p}^{\perp}$ denote the orthogonal projection. 
The Poincaré linear flow is a flow of fibered linear automorphisms $\left\{P_{t}^{X, \mathcal{R}}\right\}_{t \in \mathbb{R}}$ of the normal subbundle $E_{\mathcal{R}}^{\perp}$ defined by

$$
P_{t}^{X, \mathcal{R}}(p, \cdot)=\pi_{X_{t}(p)} \circ D X_{t}(p): E_{p}^{\perp} \rightarrow E_{X_{t}(p)}^{\perp} .
$$

Note that $P_{t}^{X, \mathcal{R}}$ is only defined on $\mathcal{R}$ and it does not extend to the whole $\Lambda$.

In [MPP] (Lemma 2.3) it was proved that the following result is a direct consequence of the volume expanding property.

Lemma 1. Let $\Lambda$ be a partially hyperbolic set of $X$ whose central direction is volume expanding. Then, there are constants $\lambda, \kappa>0$ such that for every $t>0$ and every $p \in \mathcal{R}$, we have

$$
\left\|P_{t}^{X, \mathcal{R}}(p, u)\right\| \cdot\left\|D X_{t}(p) v\right\| \geqslant \kappa e^{\lambda t}
$$

for any unit vectors $u \in F_{p}, v \in E_{p}$, where $\lambda$ is a rate of volume expansion appearing in equation (1). As a consequence, any compact invariant subset $\Lambda^{\prime} \subset \Lambda \backslash \operatorname{sing}(X \mid \Lambda)$ is hyperbolic.

\section{Proof of Theorem A}

Since $\Lambda$ is a compact partially hyperbolic set, the nonuniform contraction condition is satisfied with a constant function whose value is the number $c$ in the definition of partial hyperbolicity. Similarly, the compactness and partial hyperbolicity of $\Lambda$ imply that the angle between $E^{s}$ and any invariant subbundle of $E^{c}$ is bounded away from zero. The rest of the claims in Theorem $\mathrm{A}$ are explicitly stated in Theorem B.

Next we give a proof of Theorem B.

\section{Proof of Theorem B}

We have to show that the restriction $E_{\Lambda^{*}}^{c}$ splits in the flow direction plus a onedimensional subbundle $E_{\Lambda^{*}}^{u}$ which is nonuniformly expanded by $D X_{t}$. The subbundle $E^{u}$ will be obtained by graph transform methods as in [M] while nonuniform expansion is obtained from the volume expansion condition.

It suffices to show nonuniform hyperbolicity for the time one map of the flow $\left.X_{t}\right|_{\Lambda^{*}}$ as in [0], p. 221. Actually, we use the time minus one map and show a nonuniform contracting property instead of the nonuniform expanding one stated in (ii) of the theorem. For the sake of simplicity we use the following notation in this proof:

$$
\begin{gathered}
E=E_{\Lambda^{*}}^{X}, F=F_{\Lambda^{*}}, \\
f=\left.X_{-1}\right|_{\Lambda^{*}} \text { the inverse time one map of }\left.X_{t}\right|_{\Lambda^{*}}, \\
T(x)=\left.\operatorname{Df}(x)\right|_{E_{x}^{c}, x \in \Lambda^{*} .}
\end{gathered}
$$

Naturally $T$ defines a linear cocycle over $f$ :

$$
T(x, n)= \begin{cases}T(x, 0)=I d_{T_{x} M}, & \text { if } n=0, \\ T\left(f^{n-1}(x)\right) \circ \cdots \circ T(f(x)) \circ T(x), & \text { if } n>0, \\ T\left(f^{-n+1}(x)\right)^{-1} \circ \cdots \circ T\left(f^{-1}(x)\right)^{-1} \circ T(x)^{-1}, & \text { if } n<0 .\end{cases}
$$


We also set

$$
\begin{gathered}
T_{E}(x, n)=\left.T(x, n)\right|_{E_{x},} \\
\hat{T}(x, n)=\left(\left.\pi_{f^{n}(x)}\right|_{F_{f^{n}(x)}}\right) \circ T(x, n)=\left.P_{-n}^{X, \mathcal{R}}\right|_{F_{x}}
\end{gathered}
$$

and identify $T(x, 1)=T(x), T(x,-1)=T(x)^{-1}, \hat{T}(x, 1)=\hat{T}(x)$ and $\hat{T}(x,-1)=$ $\hat{T}(x)^{-1}$, all for $x \in \Lambda^{*}$.

We are looking for a continuous subbundle $E^{u}$ of $E_{\Lambda^{*}}^{c}$ such that $E_{\Lambda^{*}}^{c}=E \oplus E^{u}$ and $T(x, n)$ is nonuniformly contracting over $E^{u}$, since we are dealing with the time minus one map. Each $E_{x}^{u}$ will be obtained as invariant fiber of a graph transform following the arguments in the proof of Oseledec's Theorem given in $\underline{\mathrm{M}}$. In other words, we must find a continuous map $\Lambda^{*} \ni x \mapsto A(x) \in L\left(F_{x}, E_{x}\right)$ so that for $E_{x}^{u}=\operatorname{graph}(A(x))=\left\{u+A(x) u: u \in F_{x}\right\}$ we have $T(x) E_{x}^{u}=E_{f(x)}^{u}$ for every $x \in \Lambda^{*}$ and $\left.D X_{t}\right|_{E^{u}}$ satisfies the conditions in the theorem for some function $C_{\varepsilon}$ to be determined.

Write $Q(x)=T(x)-\hat{T}(x): F_{x} \rightarrow E_{f(x)}$ in such a way that

$$
T(x)(u+v)=\hat{T}(x)(u)+T(x)(v)+Q(x)(u) \text { for } u \in F_{x}, v \in E_{x} .
$$

Let $\mathbb{L}$ denote the Banach space of continuous sections of the linear bundle $L$ with fiber $L_{x}=L\left(F_{x}, E_{x}\right)$ over $\Lambda^{*}$. Define the graph transform $\Gamma: \mathbb{L} \rightarrow \mathbb{L}$ as

$$
\Gamma(A)(x)=T(f(x))^{-1} \circ A(f(x)) \circ \hat{T}(x) \text { for } A \in \mathbb{L} .
$$

Using linearity and equation (3) with $v=0$ we have, for given $A \in \mathbb{L}$ and $x \in \Lambda^{*}$,

$$
\begin{aligned}
T(x)(u+A u)= & T(x)(u)+T(x)(A(u)) \\
= & \hat{T}(x)(u)+Q(x)(u)+T(x)(A(u)) \\
= & \hat{T}(x)(u)+A(f(x)) \hat{T}(x)(u) \\
& +[T(x) A(x)(u)-A(f(x)) \hat{T}(x)(u)+Q(x)(u)] \\
= & \hat{T}(x)(u)+A(f(x)) \hat{T}(x)(u) \\
& +T(x)\left[A(x)-\Gamma(A)(x)+T(f(x))^{-1} Q(x)\right](u) .
\end{aligned}
$$

Note that the first summand on the right-hand side lies in $F_{x}$ while the last two terms belong to $E_{f(x)}$. Thus, the subspace graph $(A(x))$ is invariant by $T$ (in the sense that $\left.T(x)\left(E_{x}^{u}\right)=E_{f(x)}^{u}\right)$ if and only if

$$
A(x)-\Gamma(A)(x)=-T(f x)^{-1} \circ Q(x) .
$$

Let $B$ denote the section in $\mathbb{L}$ given by $B(x)=-T(f x)^{-1} \circ Q(x)$. We claim that the section $A \in \mathbb{L}$ defined as the formal series $\sum_{n=0}^{\infty} \Gamma^{n}(B)$ satisfies (5)). In fact,

$$
A-\Gamma(A)=\sum_{n=0}^{\infty} \Gamma^{n}(B)-\sum_{n=0}^{\infty} \Gamma^{n+1}(B)=B=-T^{-1} Q .
$$

Lemma 2 below gives a sufficient condition for the convergence of this series.

Lemma 2. There are a negative constant $\alpha$ and a continuous function $\varphi: \Lambda^{*} \rightarrow \mathbb{R}$ such that

$$
\left\|\Gamma^{n}(B)(x)\right\| \leqslant \varphi(x) e^{\alpha n}, \text { for every } n \geqslant 0, x \in \Lambda^{*} .
$$


Proof. We use Lemma 1 to estimate the rate of growth of $\hat{T}$ :

$$
\lambda_{1}(\hat{T}, x)=\limsup _{n \rightarrow+\infty} \frac{1}{n} \log \|\hat{T}(x, n)\| .
$$

To start with, inequality (2) can be written as

$$
\|\hat{T}(x, n)\| \cdot\left\|T_{E}(x, n)\right\| \leqslant \kappa \exp (-\lambda n), \text { for every } n \geqslant 1, x \in \Lambda^{*},
$$

or, in other words,

$$
\frac{1}{n} \log \|\hat{T}(x, n)\| \leqslant \frac{1}{n} \log \left(\kappa\left\|T_{E}(x, n)\right\|^{-1}\right)-\lambda, \text { for all } n \geqslant 1 .
$$

Since $T_{E}(x, n)=\left.D X_{-n}(x)\right|_{E_{x}}$ and the flow direction $E_{x}$ at $x \in \Lambda^{*}$ has zero Lyapunov exponent, we have

$$
\lim _{n \rightarrow+\infty} \frac{1}{n} \log \left(\kappa\left\|T_{E}(x, n)\right\|^{-1}\right)=0 \text { for every } x \in \Lambda^{*} .
$$

Then, taking upper limits in equation (7) we have

$$
\lambda_{1}(\hat{T}, x)=\limsup _{n \rightarrow+\infty} \frac{1}{n} \log \|\hat{T}(x, n)\| \leqslant-\lambda \quad \text { for every } x \in \Lambda^{*} .
$$

Now, for small $\varepsilon>0$ define the functions

$$
G_{\varepsilon}(x)=\sup _{n \geqslant 0} \frac{\left\|T_{E}\left(f^{n}(x),-n\right)\right\|}{\exp (n \varepsilon)} \text { and } \quad K_{\varepsilon}(x)=\sup _{n \geqslant 0} \frac{\|\hat{T}(x, n)\|}{\exp \left(n\left[\lambda_{1}(\hat{T}, x)+\varepsilon\right]\right)} .
$$

Applying definition (4) of the graph transform with $A=B$ and making obvious substitutions for the functions $G_{\varepsilon}$ and $K_{\varepsilon}$ we get

$$
\begin{aligned}
\left\|\Gamma^{n}(B)(x)\right\| & =\left\|T_{E}\left(f^{n}(x),-n\right) \circ B\left(f^{n}(x)\right) \circ \hat{T}(x, n)\right\| \\
& \leqslant\left\|B\left(f^{n}(x)\right)\right\| G_{\varepsilon}\left(f^{n}(x)\right) K_{\varepsilon}(x) \exp \left[n\left(2 \varepsilon+\lambda_{1}(\hat{T}, x)\right)\right] .
\end{aligned}
$$

Since the functions $\|Q\|$ and $\|T\|$ are bounded over $\Lambda^{*}$, we can take a uniform upper bound $M$ of $B=-T^{-1} Q$ over $\Lambda^{*}$ so that the last inequality above yields

$$
\left\|\Gamma^{n}(B)(x)\right\| \leqslant M G_{\varepsilon}\left(f^{n}(x)\right) K_{\varepsilon}(x) \exp \left[n\left(2 \varepsilon+\lambda_{1}(\hat{T}, x)\right)\right] .
$$

Using inequality (8) and arranging some factors we get from (9)

$$
\left\|\Gamma^{n}(B)(x)\right\| \leqslant M \frac{G_{\varepsilon}\left(f^{n}(x)\right)}{\exp (n \varepsilon)} K_{\varepsilon}(x) \exp [n(3 \varepsilon-\lambda] .
$$

In order to obtain a uniform estimate for $G_{\varepsilon} \circ f^{n}$ we proceed as follows. First we note that $G_{\varepsilon}$ is uniformly continuous because $T_{E}$ extends continuously to all of $\Lambda$ which is compact. This function $G_{\varepsilon}$ also has subexponential growth with respect to any $f$-invariant (in particular, $X_{t}$-invariant) probability measure with support contained in $\Lambda^{*}$ (see Lemma 11.3 in $[\mathrm{M}]$ ), i.e.,

$$
\lim _{n \rightarrow \pm \infty} \frac{1}{n} \log \left(G_{\varepsilon} \circ f^{n}(x)\right)=0
$$

for $\mu$-almost every point $x \in \Lambda$ and every $f$-invariant probability measure $\mu$. Since the periodic orbits of the flow contained in $\Lambda^{*}$ are dense in $\Lambda$, and each of them carries an ergodic invariant probability measure, it follows by continuity that

$$
\lim _{n \rightarrow \pm \infty} \frac{1}{n} \log \left(G_{\varepsilon} \circ f^{n}(x)\right)=0, \text { for every } x \in \Lambda^{*} .
$$


This fact implies that the factors

$$
\frac{G_{\varepsilon}\left(f^{n}(x)\right)}{\exp (n \varepsilon)}, n \geqslant 0
$$

in (10) are uniformly bounded in $n$ for every $x \in \Lambda^{*}$. Then we can define the real function

$$
D_{\varepsilon}(x)=\sup _{n \geqslant 0} \frac{G_{\varepsilon}\left(f^{n}(x)\right)}{\exp (n \varepsilon)}
$$

which is finite for every $x \in \Lambda^{*}$. Thus we have from (10) the following estimate for the iterates of the graph transform

$$
\left\|\Gamma^{n}(B)(x)\right\| \leqslant M D_{\varepsilon}(x) K_{\varepsilon}(x) \exp [n(3 \varepsilon-\lambda)] .
$$

Finally, take $\varepsilon>0$ small enough in such a way that the real number $\alpha=3 \varepsilon-\lambda$ is negative and define the function $\varphi(x)=M D_{\varepsilon}(x) K_{\varepsilon}(x)$ which is continuous on $\Lambda^{*}$. From this and (11), Lemma 2 follows.

Then, Lemma 2 and the Banach-Steinhaus (uniform bounding) Theorem [RN] imply that the formal series $\sum_{n=0}^{\infty} \Gamma^{n}(B)$ converges to the section $A \in \mathbb{L}$ which satisfies (5) as we proved above.

Now, for each $x \in \Lambda^{*}$ let $E_{x}^{u}$ be the graph of $A(x)$. By (15) the linear subbundle $E^{u}$ with fiber $E_{x}^{u}$ over $x \in \Lambda^{*}$ is invariant by $T=\left.D f\right|_{E_{\Lambda^{*}}}$ (and also by $D X_{t}$ ), that is,

$$
T(x)(u+A(x)(u))=\hat{T}(x)(u)+A(f(x)) \hat{T}(x)(u), \quad u \in F_{x} .
$$

Let us denote $T_{E^{u}}$ the restriction of $T$ to $E^{u}$. We proceed in the next lemma to estimate the rate of growth of $T_{E^{u}}$.

Lemma 3. The inequality

$$
\lambda_{1}\left(T_{E^{u}}, x\right) \leqslant \lambda_{1}(\hat{T}, x) \leqslant-\lambda
$$

holds for every $x \in \Lambda^{*}$, where $\lambda$ is a positive number satisfying the volume expansion property (11) for $\left.D X_{t}\right|_{E^{c}}$.

Proof. Let $\tilde{A}(x): F_{x} \rightarrow E_{x}^{u}$ denote the graph map given by $\tilde{A}(x)(u)=I_{F_{x}}(u)+$ $A(x)(u)$, where $I_{F_{x}}$ is the identity map restricted to $F_{x}$. Invariance property (12) may be expressed in terms of the map $\tilde{A}$ as

$$
T(x) \circ \tilde{A}(x)=\tilde{A}(f(x)) \circ \hat{T}(x) \text { for } x \in \Lambda^{*}
$$

which is equivalent to $T_{E^{u}}(x)=\tilde{A}(f(x)) \circ \hat{T}(x) \circ \tilde{A}(x)^{-1}$ according to the notation above. Hence, for $x \in \Lambda^{*}$, we get

$$
\begin{aligned}
\lambda_{1}\left(T_{E^{u}}, x\right)= & \limsup _{n \rightarrow+\infty} \frac{1}{n} \log \left\|T_{E^{u}}(x, n)\right\| \\
= & \limsup _{n \rightarrow+\infty} \frac{1}{n} \log \left\|\tilde{A}\left(f^{n}(x)\right) \circ \hat{T}(x, n) \circ \tilde{A}(x)^{-1}\right\| \\
\leqslant & \limsup _{n \rightarrow+\infty} \frac{1}{n} \log \left\|\tilde{A}\left(f^{n}(x)\right)\right\|+\limsup _{n \rightarrow+\infty} \frac{1}{n} \log \|\hat{T}(x, n)\| \\
& \quad+\limsup _{n \rightarrow+\infty} \frac{1}{n} \log \left\|\tilde{A}(x)^{-1}\right\|,
\end{aligned}
$$


where the last summand on the right-hand side is zero because $\left\|\tilde{A}(x)^{-1}\right\|$ does not depend on $n$, while the second one is just $\lambda_{1}(\hat{T}, x)$ which is lesser than $-\lambda$ by (8). We are left to prove that the first summand is zero also, from which we get the result. It suffices to show that $\tilde{A}$ has subexponential growth. Recalling that $A=\sum_{n \geqslant 0} \Gamma^{n}(B)$ and by Lemma 2 we obtain

$$
\left\|A\left(f^{n}(x)\right)\right\| \leqslant \sum_{n \geqslant 0}\left\|\Gamma^{n}(B)\left(f^{n}(x)\right)\right\| \leqslant \varphi\left(f^{n}(x)\right) \cdot \sum_{n \geqslant 0} e^{\alpha n}=\frac{\varphi\left(f^{n}(x)\right)}{1-e^{\alpha}} .
$$

On the other hand, we note that $\tilde{A}(x)^{-1}=\left.\pi_{x}\right|_{E_{x}^{u}}$ for every $x \in \Lambda^{*}$, where $\pi_{x}$ denotes the othogonal projection map from $E_{x}^{c}$ onto $F_{x}$, hence $\left\|\tilde{A}(x)^{-1}\right\| \leqslant 1$ and, therefore, $\|\tilde{A}(x)\| \geqslant 1$. Then, combining this with $\tilde{A}\left(f^{n}(x)\right)=I_{F_{f}(x)}+A\left(f^{n}(x)\right)$ and using triangle inequality we obtain

$$
1 \leqslant\left\|\tilde{A}\left(f^{n}(x)\right)\right\|=\left\|I_{F_{f^{n}(x)}}+A\left(f^{n}(x)\right)\right\| \leqslant 1+\left\|A\left(f^{n}(x)\right)\right\| .
$$

From this and (14) we get

$$
1 \leqslant\left\|\tilde{A}\left(f^{n}(x)\right)\right\| \leqslant 1+\frac{\varphi\left(f^{n}(x)\right)}{1-e^{\alpha}} .
$$

Now, recall that $\varphi\left(f^{n}(x)\right)=M D_{\varepsilon}\left(f^{n}(x)\right) K_{\varepsilon}\left(f^{n}(x)\right)$ where $D_{\varepsilon}$ and $K_{\varepsilon}$ are functions with subexponential growth. Then $\varphi$ has subexponential growth, and we conclude from (15) that also $\tilde{A}$ has subexponential growth as claimed. Hence, inequality (13) follows and the lemma is proved.

Continuing with the proof of the Theorem $\mathrm{B}$ in what follows we set $E_{q}^{c}=$ $E_{q} \oplus E_{q}^{u}$, where $E_{q}^{u}=\operatorname{graph}(A(q))$ is the graph of $A(q)$ for $q$ in the $f$-orbit of $x \in \Lambda^{*}$, recalling that $f=\left.X_{-1}\right|_{\Lambda^{*}}$. For this splitting we check conditions in the statement of the theorem. For this purpose we only must check the corresponding discrete versions of those conditions, that is, for $t=n \in \mathbb{Z}$ we prove the existence of $\eta>0$ and a continuous $C_{\varepsilon}$ (for given $\varepsilon>0$ small enough) so that

(a) $C_{\varepsilon}\left(f^{n}(x)\right) \leqslant e^{\varepsilon|n|} C_{\varepsilon}(x)$ for every $n \in \mathbb{Z}$;

(b) if $m, n \in \mathbb{Z}$ with $m>0$, then

$$
\left\|T\left(f^{n}(x), m\right) v\right\| \leqslant C_{\varepsilon}\left(f^{n}(x)\right) e^{-\eta m}\|v\|, \text { for } v \in E_{f^{n}(x)}^{u},
$$

(c) $\measuredangle\left(E_{f^{n}(x)}^{u}, E_{f^{n}(x)}\right) \geqslant C_{\varepsilon}\left(f^{n}(x)\right)^{-1}$ for every $n \in \mathbb{Z}$.

First note that, in general, $x \mapsto\|A(x)\|$ is not a bounded function, which means the angle $\measuredangle\left(E_{q}^{u}, E_{q}\right)$ between $E_{q}^{u}$ and $E_{q}$ is not bounded away from zero; but it always has subexponential growth. Actually,

$$
\measuredangle\left(E_{f^{n}(x)}^{u}, E_{f^{n}(x)}\right) \geqslant\left\|A\left(f^{n}(x)\right)\right\|^{-1} .
$$

By the Tempering-Kernel Lemma (S.2.12 in [KM], page 668), there is a continuous function $C_{1, \varepsilon}: \Lambda^{*} \rightarrow \mathbb{R}$ such that

$$
\|A(x)\| \leqslant C_{1, \varepsilon}(x)
$$

and

$$
e^{-\varepsilon}<\frac{C_{1, \varepsilon}(x)}{C_{1, \varepsilon}(f(x))}<e^{\varepsilon}
$$


for every $x \in \Lambda^{*}$. From (16) and (17) we obtain

$$
\measuredangle\left(E_{f^{n}(x)}^{u}, E_{f^{n}(x)}\right) \geqslant\left\|A\left(f^{n}(x)\right)\right\|^{-1} \geqslant C_{1, \varepsilon}\left(f^{n}(x)\right)^{-1},
$$

while from (18) we get by recursion that $C_{1, \varepsilon}$ satisfies

$$
C_{1, \varepsilon}\left(f^{n}(x)\right) \leqslant C_{1, \varepsilon}(x) e^{|n| \varepsilon}, \text { for all } n \in \mathbb{Z} .
$$

Then, conditions (a) and (c) above as well as (i) and (iii) in the statement of Theorem $\mathrm{B}$ hold with the function $C_{1, \varepsilon}$.

On the other hand, from Lemma 3 we have

$$
\lambda_{1}\left(T_{E^{u}}, x\right) \leqslant \lambda_{1}(\hat{T}, x) \leqslant-\lambda,
$$

which implies that for each $\varepsilon>0$ the number

$$
\sup _{m \geqslant 0} \frac{\left\|T_{E^{u}}(x, m)\right\|}{\exp [m(-\lambda+\varepsilon)]}
$$

is finite. Define the function $\tilde{C}_{\varepsilon}$ on $\Lambda^{*}$ by

$$
\tilde{C}_{\varepsilon}(x)=\sup _{m \geqslant 0} \frac{\left\|T_{E^{u}}(x, m)\right\|}{\exp [m(-\lambda+\varepsilon)]} .
$$

Fix a positive number $\eta<\lambda$ and suppose $\varepsilon>0$ small enough so that $2 \varepsilon<\lambda-\eta$. Then, given integer numbers $m, n$ with $m \geqslant 0$ we obtain

$$
\begin{aligned}
\left\|T\left(f^{n}(x), m\right) v\right\| & \leqslant \tilde{C}_{\varepsilon}\left(f^{n}(x)\right) \exp [m(-\lambda+\varepsilon)]\|v\| \\
& \leqslant \tilde{C}_{\varepsilon}\left(f^{n}(x)\right) e^{-m \varepsilon} e^{-\eta m}\|v\|
\end{aligned}
$$

for every $v \in E_{f^{n}(x)}$ and $x \in \Lambda^{*}$. Moreover, the function $\tilde{C}_{\varepsilon}$ has subexponential growth with respect to any $f$-invariant measure (see Proposition 11.2 in [M]), and arguing as in the proof of Lemma 3 it follows that this function $\tilde{C}_{\varepsilon}$ has subexponential growth on the whole $\Lambda^{*}$. Then, the Tempering-Kernel Lemma provides a function $C_{2, \varepsilon}$ so that $\tilde{C}_{\varepsilon}(x) \leqslant C_{2, \varepsilon}(x)$ and $C_{2, \varepsilon}\left(f^{n}(x)\right) \leqslant C_{2, \varepsilon}(x) e^{|n| \varepsilon}$ for every $n \in \mathbb{Z}$. In particular, $C_{2, \varepsilon}\left(f^{n}(x)\right) e^{-m \varepsilon}<C_{2, \varepsilon}\left(f^{n+m}(x)\right)$, therefore

$$
\left\|T\left(f^{n}(x), m\right) v\right\| \leqslant C_{2, \varepsilon}\left(f^{n+m}(x)\right) e^{-\eta m}\|v\| .
$$

Thus, conditions (a) and (b) above, and also the (i) and (ii) in the statement of Theorem B, hold with the function $C_{2, \varepsilon}$.

Finally, the function $C_{\varepsilon}$ defined as $C_{\varepsilon}=\max \left\{C_{1, \varepsilon}, C_{2, \varepsilon}\right\}$, satisfies simultaneously all conditions in the statement, and Theorem $\mathrm{B}$ is proved.

\section{REFERENCES}

[D] C. Doering. Persistently Transitive Vector Fields on Three-dimensional Manifolds. Dynamical Systems and Bifurcation Theory. Pitman Research Notes in Math. Series. 160, 59-89. 1987. MR0907891 (89c:58111)

[G] J. Guckenheimer. A strange strange attractor. In: The Hopf bifurcation and its applications. Marsden, J. and McCracken (Eds.). Applied Mathematical Series 9, Springer Verlag. Berlin, 1976. MR0494309 (58:13209)

[KM] A. Katok and L. Mendoza. Dynamical Systems with Nonuniform Hyperbolic Behavior. Supplement in KATOK AND Hasselblatt, Introduction to the Modern Theory of Dynamical Systems. Cambridge University Press. 1995. MR.1326374 (96c:58055)

[M] R. Mañé. Ergodic Theory and Differentiable Dynamics. Springer-Verlag. Berlin, 1987. MR0889254 (88c:58040)

[MP] C. Morales, M. J. Pacifico. Sufficient Conditions for Robustness of Attractors. Preprint at http://arxiv.org/pdf/math/.DS/0303310. 
[MPP] C. Morales, M. J. Pacifico and E. Pujals. Singular Hyperbolic Systems. Proc. Amer. Math. Soc. 127, 3393-3401. 1999. MR.1610761 (2000c:37034)

[O] V. I. Oseledec. A Multiplicative Ergodic Theorem. Lyapunov Characteristic Numbers for Dynamical Systems. Trans. Moscow Math. Soc. 19, 197-231. 1968. MR.0240280 (39:1629)

[P] Ya. Pesin. Families of Invariant Manifolds Corresponding to non-zero Characteristic Exponents. Math. USSR-Izv. 10, 1261-1305. 1976. MR0458490 (56:16690)

[PS] C. Pugh and M. Shub. Ergodic Attractors. Trans. Amer. Math. Soc. 312. 1989. MR.0983869 (90h:58057)

[RN] F. Riez and B. Nagy. Functional Analysis. Dover Publ. 1990. MR1068530 (91g:00002)

[S] Ya. Sinai. Dynamical Systems II. Encyclopedia of Mathematical Science vol. 2, Ya. G. Sinai (Ed.) Springer-Verlag. Berlin, 1989. MR1024068 (91i:58079)

[V] M. Viana. Dynamics: A Probabilistic and Geometric Perspective. Documenta Mathematica. Extra volume, ICM 1998. MR,1648047 (99h:58114)

Departamento de Matemática, Decanato de Ciencias y Tecnología, Universidad Centro Occidental Lisandro Alvarado, Apartado 400 Barquisimeto, Venezuela

E-mail address: wilmerc@uicm.ucla.edu.ve 